\title{
The Safety of Besifloxacin Ophthalmic Suspension 0.6 \% Used Three Times Daily for 7 Days in the Treatment of Bacterial Conjunctivitis
}

\author{
Ranjan Malhotra $\cdot$ Stacey Ackerman $\cdot$ \\ Lynne S. Gearinger · Timothy W. Morris • \\ Catherine Allaire
}

Published online: 19 October 2013

(c) The Author(s) 2013. This article is published with open access at Springerlink.com

\begin{abstract}
Background Besifloxacin ophthalmic suspension $0.6 \%$ (Besivance ${ }^{\circledR}$; Bausch \& Lomb, Rochester, NY, USA) was approved by the FDA in 2009 for the treatment of bacterial conjunctivitis, with a recommended 7-day dosing regimen. Objective The objective of this study was to compare the safety of besifloxacin ophthalmic suspension $0.6 \%$, administered three times a day for 7 days, with that of its vehicle. Methods This randomized, multicenter, double-masked, vehicle-controlled, parallel-group study involved 518 patients $\geq 1$ year of age with a clinical diagnosis of bacterial conjunctivitis. Patients were randomized $2: 1$ to treatment with besifloxacin $0.6 \%$ ophthalmic suspension or vehicle, one drop in the infected eye(s) TID for 7 days. Main outcomes included the incidence and types of adverse events reported by the subject or observed by the investigator at each study visit.

Results Thirty-one ocular treatment-emergent adverse events (TEAEs) were reported by 28 subjects in the study eye; 19 occurred in 17/344 (4.9\%) besifloxacin patients, and 12 occurred in $11 / 170(6.5 \%)$ vehicle patients ( $p=0.5362)$.
\end{abstract}

R. Malhotra ( ()

Ophthalmology Associates, Cornea and Laser Vision Institute, 12990 Manchester Road, Suite 200, St. Louis, MO 63131, USA e-mail: ranjan.malhotra@youreyedoc.com

\section{S. Ackerman}

Ophthalmology Associates, Philadelphia, PA, USA

L. S. Gearinger - T. W. Morris

Bausch \& Lomb, 1400 North Goodman St., Rochester, NY 14609, USA

C. Allaire

Bausch \& Lomb, 5 rue Henri Desbruères, Campus 1, Genavenir 8, 91030 Evry Cedex, France
Only two ocular events (mild instillation site reaction, one case in each group) were considered "definitely related" to study treatment. One event of self-limited dysgeusia in the besifloxacin group was considered definitely related to treatment; there were no other nonocular TEAEs considered related to treatment. There were no serious adverse events, and other safety outcomes (visual acuity, biomicroscopy, ophthalmoscopy) were unremarkable.

Conclusion These findings indicate that besifloxacin ophthalmic suspension $0.6 \%$ is safe in patients aged 1 year and older when used TID for 7 days.

\section{Introduction}

Besifloxacin ophthalmic suspension $0.6 \%$ (Besivance $^{\mathrm{TM}}$; Bausch \& Lomb, Rochester, NY, USA) was approved by the FDA in 2009 for the treatment of bacterial conjunctivitis [1]. The marketed product is formulated with DuraSite $^{\circledR}$ (InSite Vision Inc., Alameda, CA, USA), a mucoadhesive polymer delivery system designed to prolong the drug's residence time on the ocular surface, and facilitate long-acting topical antibacterial activity [2-5]. Besifloxacin is an 8-chlorofluoroquinolone that has an R7aminoazepinyl group with broad spectrum in vitro activity against a wide range of Gram-positive and Gram-negative ocular pathogens, including multidrug-resistant strains [610]. The mechanism of action of besifloxacin involves inhibition of bacterial DNA gyrase and topoisomerase IV, enzymes which are essential for the synthesis and replication of bacterial DNA [11, 12]. Unlike older fluoroquinolones, besifloxacin demonstrates relatively balanced activity against both DNA gyrase and topoisomerase IV; this minimizes the likelihood of resistance, which would require concomitant mutations in both enzymes [11, 12]. 
Three large clinical trials have established the safety and efficacy of besifloxacin ophthalmic suspension $0.6 \%$ compared with vehicle [13, 14] or active comparator (moxifloxacin ophthalmic solution $0.5 \%$ ) [15] when given three times a day for 5 days to treat acute bacterial conjunctivitis. The FDA approved labeling for besifloxacin, like most other topical ophthalmic antibacterials, recommends a 7-day treatment period for bacterial conjunctivitis [1]. Because besifloxacin exposure in the efficacy studies was limited to 5 days, the objective of this study was to compare safety outcomes associated with besifloxacin ophthalmic suspension $0.6 \%$, administered three times a day for 7 days, with those reported with the use of vehicle alone.

\section{Methods}

This study was a multicenter, randomized, double-masked, vehicle-controlled, parallel-group trial designed to evaluate the safety of besifloxacin ophthalmic suspension $0.6 \%$ compared to vehicle in patients with acute bacterial conjunctivitis. The study involved 24 investigators at 24 sites across the United States. The protocol was approved by the institutional review board at each facility, and written, informed consent was obtained for all subjects prior to enrollment. For all subjects younger than 18 years of age, signed consent was required of a legally authorized representative; subjects between the ages of 6 and 17 years also co-signed the consent forms.

The patient inclusion criteria were: age 1 year or greater; clinical diagnosis of bacterial conjunctivitis as evidenced by a minimum grade of 1 for both purulent conjunctival discharge (Scale: $0=$ absent; $1=$ mild; $2=$ moderate; $3=$ severe) and bulbar conjunctival injection (Scale: $0=$ normal; $1=$ mild; $2=$ moderate; $3=$ severe) in at least one eye; and pin-hole visual acuity (VA) equal to or better than 20/200 in both eyes (using ageappropriate VA testing). All subjects using contact lenses were instructed to discontinue contact lens wear for the entire study.

Patient exclusion criteria included: uncontrolled systemic and/or debilitating disease; known hypersensitivity to besifloxacin, fluoroquinolones, or any component of the study medication; current or expected treatment with systemic NSAIDs (exception: $\leq 81 \mathrm{mg} /$ day of acetylsalicylic acid), systemic corticosteroids, systemic antihistamines, systemic antibacterial agents; current or anticipated ocular therapy (either eye) with any ophthalmic solutions (tear substitutes, corticosteroids, NSAIDs, mast cell stabilizers, antihistamines, decongestants, antibacterial agents, immunosuppressant agents); ocular surgery (including laser surgery), either eye, within 6 weeks prior to study entry; suspected viral or allergic conjunctivitis; suspected iritis; history of recurrent corneal erosion syndrome; active ulcerative keratitis; and compromised immunity.

\subsection{Study Treatment and Follow-Up}

The subjects were randomized to treatment with besifloxacin ophthalmic suspension $0.6 \%$ or vehicle in a $2: 1$ ratio. The vehicle was identical to that used for the besifloxacin formulation without the active ingredient. Both treatments contained benzalkonium chloride $0.01 \%$.

Beginning at the first visit (Visit 1, Day 1), subjects instilled one drop of study treatment in the infected eye(s) three times daily at approximately 6-h intervals, continuing through Day 7. If patients with conjunctivitis in only one eye developed an infection in the other (fellow) eye during the study treatment period, the subject was instructed to begin using their study treatment in that eye as well. All study treatments were collected at visit 2 (Day 8). Subjects were asked to complete diary records of study treatment instillation, and medication bottles were also weighed to assess compliance. The investigators, subjects, and all other study personnel involved in the monitoring or conduct of the study were masked to the treatment received.

Cultures of the cul de sac of infected eyes were taken at each visit, before any treatment was instilled. Subjects were considered culture confirmed if the colony count (in $\mathrm{CFU} / \mathrm{mL}$ ) equaled or exceeded the threshold value on the Cagle list, as modified by Leibowitz [16]. On this list the threshold is high for species commonly found in healthy subjects' eyes (e.g., $\geq 1,000 \mathrm{CFU} / \mathrm{mL}$ for corynebacteria, $\geq 100 \mathrm{CFU} / \mathrm{mL}$ for $S$. epidermidis), but low for species that are usually not encountered (e.g., $\geq 1 \mathrm{CFU} / \mathrm{mL}$ for Pseudomonas aeruginosa), thereby reducing the likelihood of characterizing an infection as culture-confirmed due to the presence of commensal bacteria. Only one eye from each subject was designated as the study eye. Study eye determinations were made as follows: For subjects with exactly one treated eye having at least one pathogenic ocular bacterial species at or above threshold at baseline and the minimum required conjunctival discharge and bulbar conjunctival injection at baseline, the study eye was defined as that eye. For subjects with both treated eyes having at least one accepted ocular bacterial pathogen at or above threshold at baseline and the required conjunctival discharge and bulbar conjunctival injection at baseline, the study eye was defined as the treated eye with the highest combined severity of conjunctival discharge and bulbar conjunctival injection at baseline. If that combined severity was the same for both eyes, the right eye was considered the study eye. For subjects whose treated eye(s) did not have at least one accepted ocular bacterial species at or above threshold at baseline, the study eye was defined as 
the eye with the highest severity of conjunctival discharge and bulbar conjunctival injection at baseline, out of the treated eyes with the required conjunctival discharge and bulbar conjunctival injection at baseline. If the severity was the same for both eyes, the right eye was considered the study eye.

\subsection{Outcomes}

Study outcomes were assessed on Day 8 (or +1 day; Visit $2)$ and Day 11 ( \pm 1 day; Visit 3$)$.

\subsubsection{Safety}

The safety population included all subjects who received at least one dose of study medication and had at least one post-treatment safety assessment. The primary safety variable was the incidence of ocular and nonocular treatmentemergent adverse events (TEAEs). The incidence and type of TEAEs reported by the subject or observed by the investigator at each study visit were collected until study exit. For each TEAE, the investigator assessed the severity and causality with respect to treatment. Ocular TEAEs observed in baseline-designated study eyes were of primary interest and are reported here. Because treatment in fellow eyes may not have consisted of a full 7 days of exposure, those data are not included in the primary analysis. Other safety assessments included changes in visual acuity (VA) and biomicroscopy and ophthalmoscopy findings.

Age-appropriate VA testing was performed at each visit. VA was measured through a pin-hole habitual (unaided) or historical correction using a Snellen chart. For children for whom Snellen chart testing was inappropriate, the Lea Symbols or Visual Behavior (fix and follow, wince, and no wince) was used; VA measurements were attempted in all children. For any given subject, the same VA testing method was used at every study visit.

Biomicroscopy was performed at each visit to evaluate the following: hyperemia and swelling of the lids, chemosis of the conjunctiva, staining/erosion, edema, and infiltrate of the cornea, cells and flare in the anterior chamber, lens opacity, and vitreous pathology all were assessed using a 4-point scale $(0=$ None, $1=$ Mild, $2=$ Moderate , $3=$ Severe). Direct ophthalmoscopy was performed on Visits 1 and 3 to assess fundus pathology on a four-point scale $(0=$ None, $1=$ Mild, $2=$ Moderate, $3=$ Severe $)$.

\subsubsection{Efficacy}

Bacterial eradication, an objective indicator of efficacy, was evaluated in the modified Intent-to-Treat (mITT) population which included all randomized subjects from whom baseline cultures indicated bacteria levels at or above threshold for any accepted ocular bacterial pathogen. Bacterial eradication, assessed at Visits 2 and 3, was defined as the absence of all ocular bacterial species present at or above threshold at baseline. Bacterial eradication rates were determined for the mITT population overall and for the subgroup of subjects in the mITT population with baseline infections with Gram-positive species, Gram-negative species, and by most prevalent species.

In the species-specific analysis of bacterial eradication by most prevalent pathogens, fellow eyes with conjunctivitis severity meeting the study inclusion criteria that yielded baseline cultures at or above threshold for a species not present in the study eye were included.

Bacterial eradication rates were reported as observed; missing or discontinued subjects were not imputed. All microbial testing was performed at a central laboratory (Covance Central Laboratory Services, Indianapolis, IN, USA).

\subsection{Data Analysis}

\subsubsection{Sample Size Determination}

Sample size calculations determined that at least 324 subjects were needed in the besifloxacin group to provide a $>95 \%$ probability of detecting TEAEs that occur at a rate of $1 \%$, and 162 subjects were needed in the vehicle group to provide an $80 \%$ probability of detecting TEAEs that occur at a rate of $1 \%$. Assuming a $10 \%$ drop-out rate, it was planned to enroll 540 subjects to yield the minimum required total of 486 patients.

\subsubsection{Statistical Analysis}

The primary objective was to determine how the rate of TEAEs (ocular and nonocular) reported with besifloxacin ophthalmic suspension $0.6 \%$ used three times daily for 7 days compared with the rate reported with vehicle alone. Exact $95 \%$ confidence intervals were constructed around the proportion of subjects and eyes with each TEAE, and Fisher's exact test was used to test for differences between treatment groups. A similar approach was used to summarize treatment-related AEs.

\section{Results}

\subsection{Study Populations}

The safety population included 514 subjects: 344 subjects treated with besifloxacin ophthalmic suspension $0.6 \%$ and 170 subjects treated with vehicle. The mITT population 
included 299 subjects, 212 treated with besifloxacin ophthalmic suspension $0.6 \%$ and 87 treated with vehicle. In both populations, baseline demographics were similar between treatment groups (Table 1), as was ocular medical history. In the safety population, pediatric subjects ( $\leq 17$ years of age) comprised 43.0 and $35.3 \%$ of the besifloxacin and vehicle groups, respectively.

In the safety population, four subjects in the besifloxacin treatment group discontinued the study due to a TEAE [otitis media, worsening of conjunctivitis ( 2 subjects), and intervertebral disc protrusion]. All four TEAEs were considered unrelated/unlikely related to study treatment. In the vehicle group, four subjects discontinued treatment or study due to different reasons, including TEAEs: lack of efficacy and worsening of conjunctivitis, randomization error and post-traumatic pain, investigator decision and worsening of conjunctivitis, consent withdrawal and conjunctivitis. Three of these TEAEs were considered unrelated to study treatment and one was considered possibly related to study drug (lack of efficacy). Other primary reasons for discontinuation included withdrawal of consent
( $n=1$ vehicle group), lost to follow-up ( $n=1$ besifloxacin group), investigator decision ( $n=1$ besifloxacin; $n=3$ vehicle), and other reasons ( $n=3$ besifloxacin; $n=1$ vehicle).

\subsection{Compliance}

In both the mITT and safety population, the percentage of patients considered compliant (80-120\% of doses administered) was $\geq 98 \%$ in both treatment groups.

\subsection{Exposure to Study Treatment}

A total of 344 subjects were exposed to besifloxacin, while 170 subjects were exposed to vehicle (safety population). Among study eyes, mean \pm SD exposure times to study treatment were similar in the besifloxacin $(6.97 \pm 0.39$ days $)$ and vehicle $(6.92 \pm 0.52$ days $)$ treatment groups (Table 2). When considering all treated eyes (study eyes plus any treated fellow eyes), mean \pm SD exposure times were $11.42 \pm 3.43$ eye-days in the
Table 1 Baseline demographics of safety and mITT populations
mITT modified Intent to Treat population

\begin{tabular}{|c|c|c|c|c|}
\hline & \multicolumn{2}{|c|}{ Safety population } & \multicolumn{2}{|c|}{ mITT population } \\
\hline & $\begin{array}{l}\text { Besifloxacin } \\
(n=344)\end{array}$ & $\begin{array}{l}\text { Vehicle } \\
(n=170)\end{array}$ & $\begin{array}{l}\text { Besifloxacin } \\
(n=212)\end{array}$ & $\begin{array}{l}\text { Vehicle } \\
(n=87)\end{array}$ \\
\hline \multicolumn{5}{|l|}{ Age, years } \\
\hline Mean (SD) & $29.6(25.1)$ & $30.5(22.5)$ & $27.8(25.4)$ & $28.5(21.1)$ \\
\hline Range & $1-97$ & $1-92$ & $1-97$ & $1-74$ \\
\hline \multicolumn{5}{|l|}{ Distribution of age categories, $n(\%)$} \\
\hline$\geq 1-<2$ years & $19(5.5)$ & $8(4.7)$ & $19(9.0)$ & $6(6.9)$ \\
\hline $2-11$ years & $107(31.1)$ & $38(22.4)$ & $71(33.5)$ & $21(24.1)$ \\
\hline $12-17$ years & $22(6.4)$ & $14(8.2)$ & $9(4.2)$ & $5(5.7)$ \\
\hline $18-29$ years & $46(13.4)$ & $29(17.1)$ & $27(12.7)$ & $13(14.9)$ \\
\hline 30-39 years & $30(8.7)$ & $23(13.5)$ & $16(7.5)$ & $13(14.9)$ \\
\hline 40-49 years & $29(8.4)$ & $20(11.8)$ & $17(8.0)$ & $12(13.8)$ \\
\hline $50-59$ years & $38(11.0)$ & $20(11.8)$ & $20(9.4)$ & $10(11.5)$ \\
\hline$\geq 60$ years & $53(15.4)$ & $18(10.6)$ & $33(15.6)$ & $7(8.0)$ \\
\hline \multicolumn{5}{|l|}{ Sex, $n(\%)$} \\
\hline Male & $140(40.7)$ & $75(44.1)$ & $87(41.0)$ & $38(43.7)$ \\
\hline Female & $204(59.3)$ & $95(55.9)$ & $125(59.0)$ & $49(56.3)$ \\
\hline \multicolumn{5}{|l|}{ Racial background, $n(\%)$} \\
\hline American Indian/Alaskan Native & $7(2.0)$ & $3(1.8)$ & $5(2.4)$ & $1(1.1)$ \\
\hline Asian & $5(1.5)$ & $5(2.9)$ & $3(1.4)$ & $2(2.3)$ \\
\hline Black/African American & $83(24.1)$ & $40(23.5)$ & $65(30.7)$ & $30(34.5)$ \\
\hline Native Hawaiian/Pacific Islander & 0 & $1(0.6)$ & 0 & 0 \\
\hline White & $210(61.0)$ & $102(60.0)$ & $121(57.1)$ & $49(56.3)$ \\
\hline Other & $39(11.3)$ & $19(11.2)$ & $18(8.5)$ & $5(5.7)$ \\
\hline \multicolumn{5}{|l|}{ Ethnicity, $n(\%)$} \\
\hline Not Hispanic and Not Latino & $194(56.4)$ & $101(59.4)$ & $126(59.4)$ & $58(66.7)$ \\
\hline Hispanic or Latino & $150(43.6)$ & $69(40.6)$ & $86(40.6)$ & $29(33.3)$ \\
\hline
\end{tabular}


besifloxacin treatment group and $11.56 \pm 3.38$ eye-days in the vehicle treatment group.

\subsection{Ocular Treatment-Emergent Adverse Events (TEAEs)}

Overall, 31 ocular TEAEs were reported by 28 subjects in the study eye (Table 3), with no significant difference noted between treatment groups. In the besifloxacin group, 19 events were reported in $17 / 344(4.9 \%)$ patients; 12 events were reported in 11/170 (6.5\%) vehicle patients $(p=0.5362)$. Only two ocular events (one case of instillation site reaction in each of the besifloxacin and vehicle groups) were considered "definitely related" to study treatment by the investigator; these events were both considered mild and resolved without treatment. No subjects were removed from the study due to these events. One event of conjunctivitis in the vehicle group was considered "probably related" to treatment. Four TEAEs (punctate keratitis, instillation site erythema, instillation site pain, and instillation site reaction) in the besifloxacin group were considered "possibly related" to treatment, while four TEAEs (conjunctivitis, conjunctival edema, punctate keratitis, and instillation site irritation) were considered "possibly related" to treatment in the vehicle group. All study eye ocular events in the besifloxacin group were considered mild or moderate in severity, while one event (conjunctivitis) in the vehicle group was considered severe.

Ocular TEAE reported in fellow treated eyes were similar to those reported in study eyes with 21 events reported in 18/220 (8.2\%) besifloxacin treated patients and 11 events reported in 11/115 (9.6\%) vehicle treated patients $(p=0.6855)$. Consistent with study eyes, one case of instillation site reaction in each treatment group was considered "definitely related" to study treatment. Further, three ocular TEAEs (punctate keratitis, instillation site erythema, and instillation site reaction) in the besifloxacin group and two TEAEs (conjunctivitis and instillation site irritation) in the vehicle group were considered "possibly related" to treatment. All ocular TEAEs in the fellow treated eyes were considered mild or moderate in severity.

Table 2 Exposure to study treatment (safety population-study eyes)

\begin{tabular}{lll}
\hline $\begin{array}{l}\text { Number of } \\
\text { eye days }\end{array}$ & $\begin{array}{l}\text { Besifloxacin, } \\
n(\%)(N=344)\end{array}$ & $\begin{array}{l}\text { Vehicle, } n(\%) \\
(N=170)\end{array}$ \\
\hline$\leq 6$ & $8(2.3 \%)$ & $5(2.9 \%)$ \\
7 & $332(96.5 \%)$ & $164(96.5 \%)$ \\
$8-11$ & $4(1.2 \%)$ & $1(0.6 \%)$ \\
$\geq 12$ & 0 & 0 \\
Mean \pm SD eye days & $6.97 \pm 0.39$ & $6.92 \pm 0.52$ \\
\hline
\end{tabular}

3.5 Nonocular Treatment-Emergent Adverse Events (TEAEs)

Overall, 16 nonocular TEAEs were reported by 15 subjects (Table 4), including 10 events in 9/344 (2.6\%) besifloxacin subjects and six events in 6/170 (3.5\%) vehicle subjects; there was no significant difference in the incidence of nonocular TEAEs between the two treatment groups $(p=0.5837)$. One nonocular event (mild dysgeusia in the besifloxacin group) was considered definitely related to treatment; this event resolved without treatment, and the subject was not discontinued from the study. All other nonocular events were considered unrelated or unlikely related to study treatment. No serious AEs were reported or observed.

\subsection{Visual Acuity (VA)}

No subject in either treatment group had a reduction in VA by more than two lines at any visit. Most subjects showed either an improvement or no change from baseline at Visit 2 (92.1\%, besifloxacin; $96.6 \%$ vehicle) and Visit 3 (93.7\%, besifloxacin; $95.2 \%$, vehicle). VA findings were similar for treated fellow eyes.

\subsection{Biomicroscopy}

Overall, very few subjects $(<2 \%$ in either treatment group) presented treatment emergent biomicroscopy findings in the study eye at any visit. There were no significant differences noted between treatment groups for the frequency of any biomicroscopy findings at Day 8 [6 $(1.8 \%)$ besifloxacin subjects vs. $3(1.8 \%)$ vehicle subjects] or Day 11 [3 $(0.9 \%)$ besifloxacin subjects vs. 0 vehicle subjects]. Findings were similar for treated fellow eyes. Likewise, there were no significant differences between treatment groups for the specific slit lamp evaluations of the eyelid, conjunctiva, cornea, anterior chamber, lens, or vitreous.

\subsection{Ophthalmoscopy}

There were no treatment emergent ophthalmoscopy findings on Day 11 in either the study eyes or treated fellow eyes for either treatment group.

\subsection{Bacterial Eradication (Efficacy)}

\subsubsection{Overall}

As expected, at Visit 2 (Day 8), besifloxacin-treated study eyes had a higher rate of bacterial eradication 
Table 3 Ocular treatmentemergent adverse events (TEAEs) by investigator assessment of relationship to study medication (study eye only, safety population) a Includes events considered by investigator as "possibly", "probably", or "definitely" related; events with unknown relationship were counted as "probably related"

\begin{tabular}{|c|c|c|c|c|}
\hline & \multicolumn{2}{|c|}{ Besifloxacin $0.6 \%(N=344)$} & \multicolumn{2}{|c|}{ Vehicle $(N=170)$} \\
\hline & $\begin{array}{l}\text { Unlikely or } \\
\text { unrelated }\end{array}$ & Related $^{\mathrm{a}}$ & $\begin{array}{l}\text { Unlikely or } \\
\text { unrelated }\end{array}$ & Related $^{\mathrm{a}}$ \\
\hline Total number of TEAEs & 14 & 5 & 6 & 6 \\
\hline Number of subjects with at least 1 TEAE & $13(3.8 \%)$ & $4(1.2 \%)$ & $6(3.5 \%)$ & $5(2.9 \%)$ \\
\hline Conjunctivitis & $3(0.9 \%)$ & 0 & $1(0.6 \%)$ & $2(1.2 \%)$ \\
\hline Eyelid erythema & $2(0.6 \%)$ & 0 & 0 & 0 \\
\hline Blepharitis & $1(0.3 \%)$ & 0 & $1(0.6 \%)$ & 0 \\
\hline Corneal infiltrates & $1(0.3 \%)$ & 0 & 0 & 0 \\
\hline Dacryocystitis & $1(0.3 \%)$ & 0 & 0 & 0 \\
\hline Eye pain & $1(0.3 \%)$ & 0 & 0 & 0 \\
\hline Lacrimation increased & $1(0.3 \%)$ & 0 & 0 & 0 \\
\hline Conjunctival hemorrhage & $1(0.3 \%)$ & 0 & 0 & 0 \\
\hline Conjunctival edema & $1(0.3 \%)$ & 0 & 0 & $1(0.6 \%)$ \\
\hline Conjunctivitis, allergic & 0 & 0 & $1(0.6 \%)$ & 0 \\
\hline Punctate keratitis & 0 & $1(0.3 \%)$ & 0 & $1(0.6 \%)$ \\
\hline Scleritis & 0 & 0 & $1(0.6 \%)$ & 0 \\
\hline Instillation site pain/irritation/erythema & 0 & $2(0.6 \%)$ & 0 & $1(0.6 \%)$ \\
\hline Instillation site reaction & 0 & $2(0.6 \%)$ & 0 & $1(0.6 \%)$ \\
\hline Pain & 0 & 0 & $1(0.6 \%)$ & 0 \\
\hline Herpes dermatitis & $1(0.3 \%)$ & 0 & 0 & 0 \\
\hline Post-traumatic pain & 0 & 0 & $1(0.6 \%)$ & 0 \\
\hline Corneal staining & $1(0.3 \%)$ & 0 & 0 & 0 \\
\hline
\end{tabular}

Table 4 Nonocular treatmentemergent adverse events (TEAEs) by investigator assessment of relationship to study medication (safety population)

\footnotetext{
a Includes events considered by investigator as "possibly", "probably", or "definitely" related; events with unknown relationship were counted as "probably related"
}

\begin{tabular}{|c|c|c|c|c|}
\hline & \multicolumn{2}{|c|}{ Besifloxacin $0.6 \%(N=344)$} & \multicolumn{2}{|c|}{ Vehicle $(N=170)$} \\
\hline & $\begin{array}{l}\text { Unlikely or } \\
\text { unrelated }\end{array}$ & Related $^{\mathrm{a}}$ & $\begin{array}{l}\text { Unlikely or } \\
\text { unrelated }\end{array}$ & Related $^{\mathrm{a}}$ \\
\hline Total number of TEAEs & 9 & 1 & 6 & 0 \\
\hline Number of subjects with at least 1 TEAE & $8(2.3 \%)$ & $1(0.3 \%)$ & $6(3.5 \%)$ & 0 \\
\hline Ear pain & $1(0.3 \%)$ & 0 & 0 & 0 \\
\hline Dysgeusia & 0 & $1(0.3 \%)$ & 0 & 0 \\
\hline Pyrexia & $1(0.3 \%)$ & 0 & 0 & 0 \\
\hline Nasopharyngitis & $2(0.6 \%)$ & 0 & $1(0.6 \%)$ & 0 \\
\hline Otitis media & $1(0.3 \%)$ & 0 & 0 & 0 \\
\hline Upper respiratory tract infection & $1(0.3 \%)$ & 0 & 0 & 0 \\
\hline Bronchitis & 0 & 0 & $1(0.6 \%)$ & 0 \\
\hline Gastroenteritis, viral & 0 & 0 & $1(0.6 \%)$ & 0 \\
\hline Intervertebral disc protrusion & $1(0.3 \%)$ & 0 & 0 & 0 \\
\hline Cyst & 0 & 0 & $1(0.6 \%)$ & 0 \\
\hline Headache & $1(0.3 \%)$ & 0 & $1(0.6 \%)$ & 0 \\
\hline Nasal congestion & $1(0.3 \%)$ & 0 & 0 & 0 \\
\hline Rhinitis, allergic & 0 & 0 & $1(0.6 \%)$ & 0 \\
\hline
\end{tabular}

than vehicle-treated study eyes [83.5\% (172/206) vs. $45.0 \%$ (36/80), respectively; Fig. 1a]. A similar pattern was observed at Day 11, although the difference between the groups was smaller $[84.5 \%(169 / 200)$ vs. $57.8 \%(48 / 83)]$.

\subsubsection{Eradication of Bacterial Species According to Gram Stain}

Bacterial eradication by baseline infection with either Gram-positive or Gram-negative species did not differ 
significantly from overall species. For infections caused by Gram-positive bacterial species (Fig. 1b), besifloxacintreated eyes had a higher rate of bacterial eradication in the study eye at both Visit 2 and Visit 3 compared to vehicletreated eyes. At Visit 2 (Day 8), $82.8 \%$ (135/163) of besifloxacin-treated eyes had bacterial eradication compared to $38.3 \%(23 / 60)$ of vehicle-treated eyes. At Visit 3 (Day 11), $84.3 \%(134 / 159)$ of besifloxacin-treated eyes had bacterial eradication compared to $54.8 \%(34 / 62)$ of vehicle-treated eyes. For Gram-negative bacterial species (Fig. 1c), besifloxacin-treated eyes also had higher rates of bacterial eradication at both Visit 2 and Visit 3 than vehicle-treated eyes. At Visit 2 (Day 8), $91.1 \%$ (72/79) of besifloxacin-treated eyes had bacterial eradication compared to $71.4 \%(20 / 28)$ of vehicle-treated eyes. At Visit 3 (Day 11), $89.6 \%$ (69/77) of besifloxacin-treated eyes had bacterial eradication compared to $75.9 \%(22 / 29)$ of vehicle-treated eyes.
Results for bacterial eradication for Gram-positive and Gram-negative bacterial species in the treated fellow eyes were similar to those for study eyes; besifloxacin-treated subjects had a higher rate of overall bacterial eradication in fellow eyes at both Visit 2 and Visit 3 than vehicle-treated subjects (data not shown).

\subsubsection{Eradication of Most Prevalent Species}

A total of 528 pathogens were isolated from culture confirmed eyes at baseline. The most common species isolated were Staphylococcus epidermidis (22.0\%), followed by Haemophilus influenzae (16.7\%), Staphylococcus aureus $(13.1 \%)$, Streptococcus mitis group (10.4\%) and Streptococcus pneumoniae $(5.1 \%)$. In the analysis of bacterial eradication by baseline infection with these species bacterial eradication rates were higher with besifloxacin ophthalmic suspension compared with vehicle with the
Fig. 1 Bacterial eradication rates in besifloxacin- and vehicle-treated baselinedesignated study eyes following TID treatment for 7 days (modified ITT population). Data shown for a overall bacterial species, b Gram-positive species, and $\mathbf{c}$ Gram-negative species

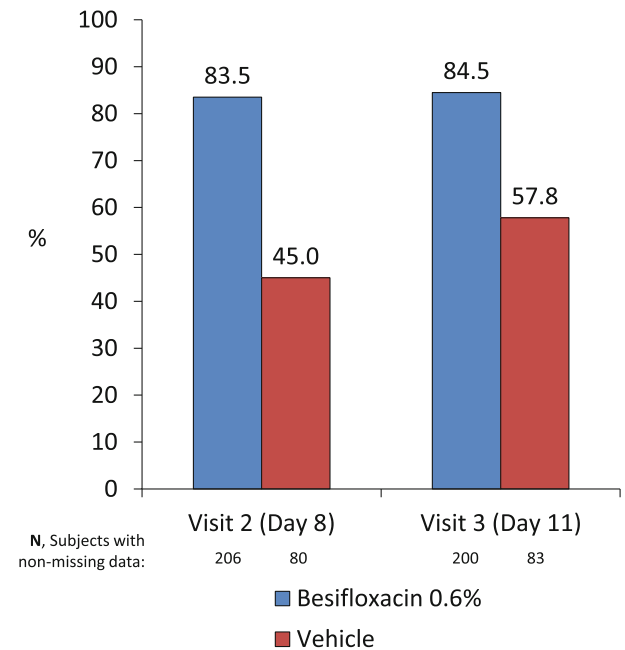

(a) Over all

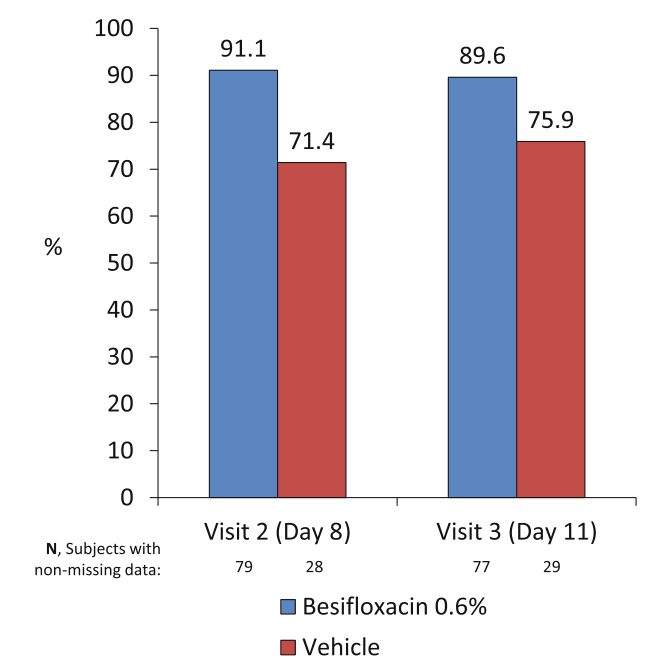

(c) Gram-Negative Species

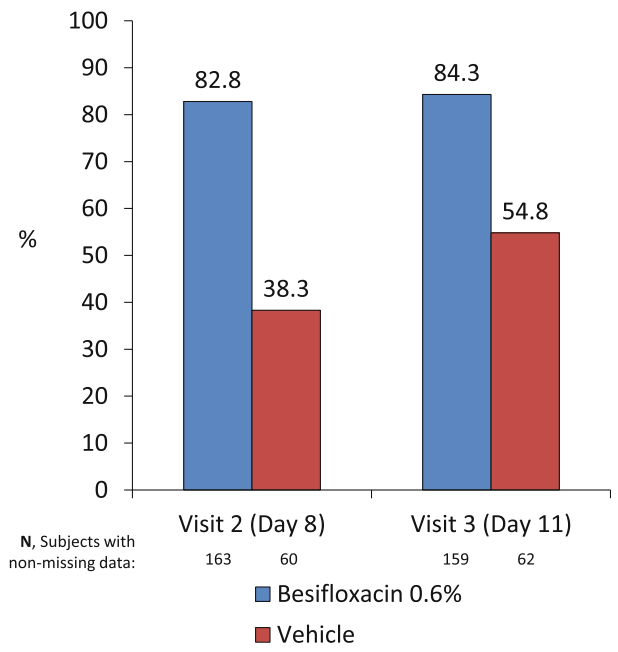

(b) Gram-Positive Species 
exception of Visit 2 for $S$. pneumoniae and $S$. mitis group likely due to the small sample size. Figure 2 presents bacterial eradication by baseline infection for the four most prevalent pathogens.

\section{Discussion}

Results from this large, randomized, double-masked, vehicle-controlled study, which included 518 subjects from 24 sites across the USA, provides evidence of the safety of besifloxacin given three times daily for 7 days in the treatment of bacterial conjunctivitis. The incidences of nonocular TEAEs and study eye ocular TEAEs were low and occurred at similar rates for besifloxacin-treated and vehicle-treated subjects. Ocular events considered at least possibly related to treatment were reported by only $1.2 \%$ of besifloxacin-treated subjects and $2.9 \%$ of vehicle-treated subjects; almost all ocular events were mild or moderate and self-limited. There were no serious adverse events, and other safety outcomes (visual acuity, biomicroscopy, ophthalmoscopy) were unremarkable.

While topical administration of besifloxacin ophthalmic suspension produces high and prolonged ocular surface drug concentrations, a previous study found that the average systemic concentration of besifloxacin after repeated three times daily dosing was less than $0.5 \mathrm{ng} / \mathrm{mL}$ [17]. This suggests that the risk of systemic side effects after topical administration of besifloxacin ophthalmic suspensions is very low. In fact, there was only one nonocular AE (dysgeusia) in the present study that was considered even possibly related to treatment (besifloxacin-treated group).

The safety results of this 7-day study are consistent with previous tolerability findings from three independent studies of besifloxacin ophthalmic suspension given three times daily for 5 days [13-15]. A pooled analysis of safety data from these three clinical studies reported that the most commonly reported ocular adverse events in besifloxacintreated patients were, in order of frequency, blurred vision $(2.1 \%)$, eye pain $(1.8 \%)$, eye irritation (1.4\%), conjunctivitis $(1.2 \%)$, and eye pruritus (1.1\%) [18]. Blurred vision, eye irritation, and conjunctivitis were reported significantly less frequently by besifloxacin-treated patients than by patients given vehicle [18]. In the study comparing besifloxacin and moxifloxacin, eye irritation was significantly less common for besifloxacin-treated eyes $(0.3 \%)$ than in moxifloxacin-treated eyes $(1.4 \% ; p=0.02)$ [15]. Commonly reported adverse effects with other topical fluoroquinolones include stinging, chemosis, local irritation, superficial punctate keratitis, and conjunctival hyperemia, although more serious events are possible [19]. Overall, the safety results for besifloxacin are comparable,
Fig. 2 Bacterial eradication rates in species-specific study eyes following TID treatment for 7 days with besifloxacin ophthalmic suspension $0.6 \%$ (solid lines) or vehicle (dashed lines) (modified ITT population). (data shown by most prevalent species)
S. epidermidis

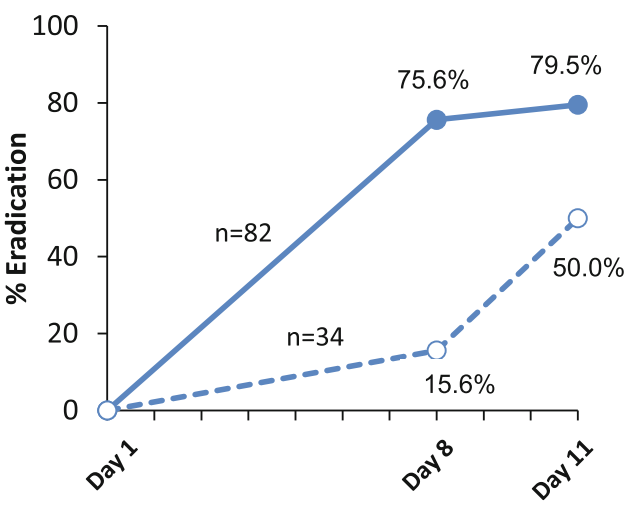

S. aureus

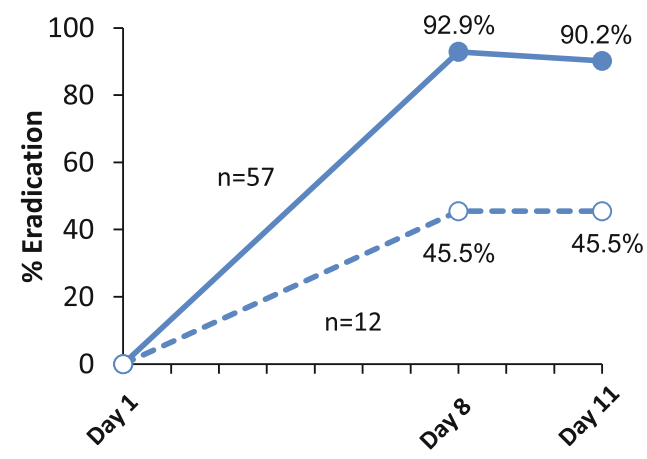

H. influenzae

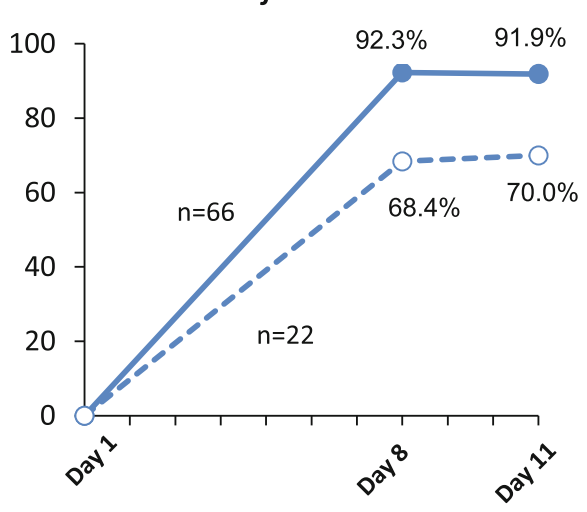

S. mitis group

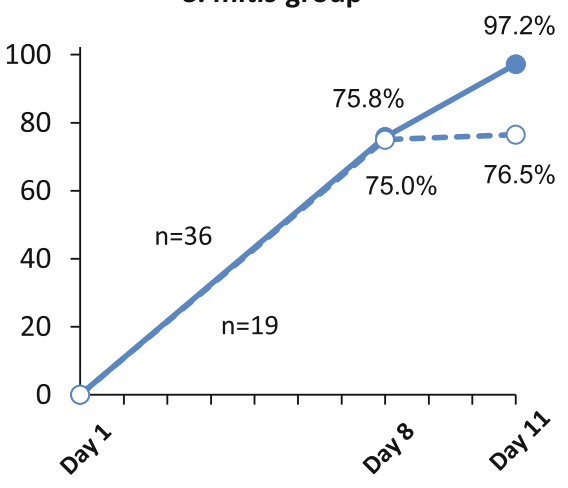


though no serious events were observed in the present study.

Also consistent with previous studies, bacterial eradication was seen at a higher rate in besifloxacin-treated eyes than in vehicle-treated eyes at Day 8 and Day 11, though the difference between the groups was smaller at Day 11 . This outcome is not unexpected, given the natural course of the disease. Acute bacterial conjunctivitis is known to be self-limited in most cases, resolving spontaneously due to the host's immune factors in 1-2 weeks [20]. However, topical ophthalmic antibiotics are warranted as they contribute to hastening clinical resolution and microbiological remission, decreasing the risk of relapse and the development of complications such as keratitis, orbital cellulitis, and panophthalmitis [21]. A meta-analysis of studies in which topical antibiotic treatment was compared to placebo in the management of bacterial conjunctivitis demonstrated that topical antibiotics were of most benefit in improving early (Days 2-5) clinical and microbiological remission rates as opposed to later clinical and microbiological remission rates (6-10 days) [21]. The treatment effect (difference between active and vehicle) with besifloxacin ophthalmic suspension $0.6 \%$ noted at Day 8 in this study was within the range reported in other studies of topical antibiotics in the treatment of bacterial conjunctivitis, or 15-39\% at Day 6-10 [22].

In previous studies of besifloxacin ophthalmic suspension $0.6 \%$ administered TID for 5 days in the treatment of bacterial conjunctivitis, eradication rates were already very high at Day 4/5 (91.5\% for besifloxacin vs. $59.7 \%$ for vehicle [14]; $93.3 \%$ for besifloxacin vs. $91.1 \%$ for moxifloxacin [15]; and $90.0 \%$ for besifloxacin vs. $46.6 \%$ for vehicle [13], demonstrating the rapid effect of besifloxacin treatment; these bacterial eradication rates were also associated with rapid improvements in the clinical signs and symptoms of acute bacterial conjunctivitis. It follows that although the earliest time point of bacterial eradication assessment in this study was Day 8, it is likely that high bacterial eradication rates were achieved much earlier.

In the present study, similar bacterial eradication rates were seen at Days 8 and 11 for Gram-positive (82.8 and $84.3 \%$, respectively) and Gram-negative species (91.1 and $89.6 \%$, respectively) in besifloxacin-treated eyes. Bacterial eradication rates with vehicle were lower on Days 8 and 11 for both Gram-positive (38.3 and 54.8\%, respectively) and Gram-negative species (71.4 and $75.9 \%)$. The most common bacterial species isolated at baseline in order of prevalence were $S$. epidermidis, $H$. influenzae, $S$. aureus, and $S$. mitis group. As expected, bacterial eradication rates for these species also appeared better with besifloxacin treatment compared with vehicle treatment.
It deserves mention that the besifloxacin ophthalmic suspension $0.6 \%$ formulation contains the preservative benzalkonium chloride (BAK) at a concentration of $0.01 \%$. The presence of BAK in topical ophthalmic formulations has been shown to have dose-dependent conjunctival and corneal epithelial cell toxicity [23-26], although the clinical relevance of this phenomenon in routine clinical practice, especially with short-term usage, is not yet clear. The very low rate of adverse effects noted in the current study does not suggest any toxicity risk with the concentration of BAK present in the besifloxacin suspension formulation. BAK has also been shown to possess inherent bacteriostatic and bactericidal activities [27, 28]; thus, it is possible that BAK contributed to the bacterial eradication rate observed in both the besifloxacin treatment group and vehicle group in the present study, as both treatments contained $\mathrm{BAK}$ at a concentration of $0.01 \%$. Since the present study did not include an additional control group without BAK, any possible confounding of bacterial eradication rates from the inclusion of BAK cannot be fully evaluated.

In conclusion, the results of this analysis expand upon those previously identified using besifloxacin ophthalmic suspension $0.6 \%$ for 5 days. These new data indicate that besifloxacin ophthalmic suspension $0.6 \%$ is safe for use in patients aged 1 year and older with bacterial conjunctivitis when used TID for 7 days, while providing high bacterial eradication rates.

Acknowledgements This study was sponsored by Bausch \& Lomb Incorporated (Rochester, NY, USA). Clinical monitoring and clinical trial supplies were provided by Bausch \& Lomb. The authors thank Howard M. Proskin \& Associates, Inc. and Lening Zhang, PhD, of Bausch \& Lomb for statistical analysis of the data. Publication was sponsored by Bausch \& Lomb, with editorial assistance provided by Churchill Communications.

Open Access This article is distributed under the terms of the Creative Commons Attribution Noncommercial License which permits any noncommercial use, distribution, and reproduction in any medium, provided the original author(s) and the source are credited.

\section{References}

1. Besivance [package insert]. Rochester: Bausch \& Lomb Inc (2009).

2. Protzko E, Bowman L, Abelson M, for the AzaSite Clinical Study Group, et al. Phase 3 safety comparisons for $1.0 \%$ azithromycin in polymeric mucoadhesive eye drops versus $0.3 \%$ tobramycin eye drops for bacterial conjunctivitis. Invest Ophthalmol Vis Sci. 2007;48:3425-9.

3. Bowman LM, Si E, Pang J, Archibald R, Friedlaender M. Development of a topical polymeric mucoadhesive ocular delivery system for azithromycin. J Ocul Pharmacol Ther. 2009;25(2):133-9. 
4. Akpek EK, Vittitow J, Verhoeven RS, et al. Ocular distribution and pharmacokinetics of a novel ophthalmic 1\% azithromycin formulation. J Ocul Pharmacol Ther. 2009;25(5):433-9.

5. Si EC, Bowman LM, Hosseini K. Pharmacokinetic comparisons of bromfenac in DuraSite and Xibrom. J Ocul Pharmacol Ther. 2011;27(1):61-6.

6. Haas W, Gearinger LS, Usner DW, et al. Integrated analysis of three bacterial conjunctivitis trials of besifloxacin ophthalmic suspension, $0.6 \%$ : etiology of bacterial conjunctivitis and antibacterial susceptibility profile. Clin Ophthalmol. 2011;5: 1369-79.

7. Ward KW, Lepage J-F, Driot J-Y. Nonclinical pharmacodynamics, pharmacokinetics, and safety of BOL-3032243-A, a novel fluoroquinolone antimicrobial agent for topical ophthalmic use. J Ocul Pharmcol Ther. 2007;23:243-56.

8. Haas W, Pillar CM, Zurenko GE, et al. Besifloxacin, a novel fluoroquinolone, has broad-spectrum in vitro activity against aerobic and anaerobic bacteria. Antimicrob Agents Chemother. 2009;53:3552-60.

9. Haas W, Pillar C, Hesje CK, Sanfilippo CM, Morris TW. Bactericidal activity of besifloxacin against staphylococci, Streptococcus pneumoniae and Haemophilus influenzae. J Antimicrob Chemother. 2010;65:1441-7.

10. Haas W, Pillar CM, Torres M, Morris TW, Sahm DF. Monitoring antibiotic resistance in ocular microorganisms: Results from the ARMOR 2009 Surveillance Study. Am J Ophthalmol. 2011;152: 567-74.

11. Cambau E, Matrat S, Xiao-Su P, et al. Target specificity of the new fluoroquinolone besifloxacin in Streptococcus pneumoniae, Staphylococcus aureus and Escherichia coli. J Antimicrob Chemother. 2009;63:443-50.

12. Sanfilippo CM, Hesje C, Haas W, Morris TW. Topoisomerase mutations that are associated with high-level resistance to earlier fluoroquinolones in Staphylococcus aureus have less effect on the antibacterial activity of besifloxacin. Chemotherapy. 2011;57: 363-71.

13. Karpecki P, DePaolis M, Hunter JA, et al. Besifloxacin ophthalmic suspension $0.6 \%$ in patients with bacterial conjunctivitis: a multicenter, prospective, randomized, double-masked, vehiclecontrolled, 5-day efficacy and safety study. Clin Ther. 2009;31:514-26.

14. Tepedino ME, Heller WH, Usner DW, et al. Phase III efficacy and safety study of besifloxacin ophthalmic suspension $0.6 \%$ in the treatment of bacterial conjunctivitis. Curr Med Res Opin. 2009;25:1159-69.
15. McDonald MB, Protzko EE, Brunner LS, et al. Efficacy and safety of besifloxacin ophthalmic suspension $0.6 \%$ compared with moxifloxacin ophthalmic solution $0.5 \%$ for treating bacterial conjunctivitis. Ophthalmology. 2009;116:1615-23.

16. Leibowitz HM. Antibacterial effectiveness of ciprofloxacin $0.3 \%$ ophthalmic solution in the treatment of bacterial conjunctivitis. Am J Ophthalmol. 1991;112(Suppl):29S-33S.

17. Proksch JW, Granvil CP, Siou-Mermet R, et al. Ocular pharmacokinetics of besifloxacin following topical administration to rabbits, monkeys, and humans. J Ocul Pharmacol Ther. 2009;25:335-44.

18. Comstock TL, Paterno MR, DeCory HH, Usner DW. Safety and tolerability of besifloxacin ophthalmic suspension $0.6 \%$ in the treatment of bacterial conjunctivitis: data from six clinical and Phase I safety studies. Clin Drug Investig. 2010;30:675-85.

19. Thompson AM. Ocular toxicity of fluoroquinolones. Clin Exp Ophthalmol. 2007;35:566-77.

20. Gunnar H. Acute bacterial conjunctivitis. Acta Ophthalmol. 2008;86:5-17.

21. Sheikh A, Hurwitz B. Antibiotics versus placebo for acute bacterial conjunctivitis (review). Cochrane Database Syst Rev. 2006;2:CD001211.

22. DeLeon J, Silverstein BE, Allaire C, et al. Besifloxacin ophthalmic suspension $0.6 \%$ administered twice daily for 3 days in the treatment of bacterial conjunctivitis in adults and children. Clin Drug Investig. 2012;32(5):303-17.

23. Meloni M, Cattaneo G, De Servi B. Corneal epithelial toxicity of antiglaucoma formulations: in vitro study of repeated applications. Clin Ophthalmol. 2012;6:1433-40.

24. Whitson JT, Petroll WM. Corneal epithelial cell viability following exposure to ophthalmic solutions containing preservatives and/or antihypertensive agents. Adv Ther. 2012;29:874-88.

25. Labbé A, Pauly A, Liang H, et al. Comparison of toxicological profiles of benzalkonium chloride and polyquaternium-1: an experimental study. J Ocul Pharmacol Ther. 2006;22:267-78.

26. Sarkar J, Chaudhary S, Namavari A, et al. Corneal toxicity due to topical benzalkonium chloride. Invest Ophthalmol Vis Sci. 2012;53:1792-802.

27. McDonnell G, Russell AD. Antiseptics and disinfectants: activity, action, and resistance. Clin Microb Rev. 1999;12:147-79.

28. Blondeau JM, Boros S, Hesje CK. Antimicrobial efficacy of gatifloxacin and moxifloxacin with and without benzalkonium chloride compared with ciprofloxacin and levofloxacin against methicillin-resistant Staphylococcus aureus. J Chemother. 2007;19:146-51. 